\title{
Article \\ Sharp Upper and Lower Bounds of VDB Topological Indices of Digraphs
}

\author{
Juan Monsalve (i) and Juan Rada *(1) \\ Instituto de Matemáticas, Universidad de Antioquia, Calle 67 No. 53-108, Medellín 050010, Colombia; \\ daniel.monsalve@udea.edu.co \\ * Correspondence: pablo.rada@udea.edu.co
}

Citation: Monsalve, J.; Rada, J. Sharp Upper and Lower Bounds of VDB Topological Indices of Digraphs. Symmetry 2021, 13, 1903. https:// doi.org/10.3390/sym13101903

Academic Editors: Jose M. Rodriguez and Eva Tourís

Received: 7 September 2021

Accepted: 2 October 2021

Published: 9 October 2021

Publisher's Note: MDPI stays neutral with regard to jurisdictional claims in published maps and institutional affiliations.

Copyright: (c) 2021 by the authors. Licensee MDPI, Basel, Switzerland. This article is an open access article distributed under the terms and conditions of the Creative Commons Attribution (CC BY) license (https:// creativecommons.org/licenses/by/ $4.0 /)$.
Abstract: A vertex-degree-based (VDB, for short) topological index $\varphi$ induced by the numbers $\left\{\varphi_{i j}\right\}$ was recently defined for a digraph $D$, as $\varphi(D)=\frac{1}{2} \sum_{u v} \varphi_{d_{u}^{+} d_{v}^{-}}$, where $d_{u}^{+}$denotes the out-degree of the vertex $u, d_{v}^{-}$denotes the in-degree of the vertex $v$, and the sum runs over the set of $\operatorname{arcs} u v$ of $D$. This definition generalizes the concept of a VDB topological index of a graph. In a general setting, we find sharp lower and upper bounds of a symmetric VDB topological index over $\mathcal{D}_{n}$, the set of all digraphs with $n$ non-isolated vertices. Applications to well-known topological indices are deduced. We also determine extremal values of symmetric VDB topological indices over $\mathcal{O T}(n)$ and $\mathcal{O}(G)$, the set of oriented trees with $n$ vertices, and the set of all orientations of a fixed graph $G$, respectively.

Keywords: vertex-degree-based topological index; digraph; orientation of a graph; extremal value

MSC: 05C92; 05C09; 05C35

\section{Introduction}

A digraph $D$ is a finite nonempty set $V$ called vertices, together with a set $A$ of ordered pairs of distinct vertices of $D$, called arcs. If $a=(u, v)$ is an arc of $D$, then we write $u v$ and say that the two vertices are adjacent. Given a vertex $u$ of $G$, the out-degree of $u$ is denoted by $d_{u}^{+}$and defined as the number of arcs of the form $u v$, where $v \in V$. The in-degree of $u$ is denoted by $d_{u}^{-}$and defined as the number of arcs of the form $w u$, where $w \in V$. A vertex $u$ in $D$ is called a sink vertex (resp. source vertex) if $d_{u}^{+}=0$ (resp. $d_{u}^{-}=0$ ). We denote by $q=q(D)$ the number of vertices of $D$ which are sink vertices or source vertices. If $d_{u}^{+}=d_{u}^{-}=0$, then $u$ is an isolated vertex. The set of digraphs with $n$ non-isolated vertices is denoted by $\mathcal{D}_{n}$.

One special class of digraphs is the oriented graphs. A pair of arcs of a digraph $D$ of the form $u v$ and $v u$ are called symmetric arcs. If $D$ has no symmetric arcs, then $D$ is an oriented graph. We note that $D$ can be obtained from a graph $G$ by substituting each edge $u v$ by an arc $u v$ or $v u$, but not both. In this case, we say that $D$ is an orientation of G. For example, in Figure 1 we show the directed path $\vec{P}_{n}$ and the directed cycle $\vec{C}_{n}$, orientations of the path $P_{n}$ and cycle $C_{n}$, respectively. A sink-source orientation of a graph $G$ is an orientation in which every vertex is a sink vertex or a source vertex. Clearly, when we reverse the orientations of all arcs in a sink-source orientation, we obtain a sink-source orientation again. For instance, the digraphs $\vec{K}_{1, n-1}$ and $\vec{K}_{n-1,1}$ in Figure 1 are sink-source orientations of the star $S_{n}$. Note that $\vec{K}_{n-1,1}$ is obtained by reversing all arcs of $\vec{K}_{1, n-1}$.

Let $D_{1}=\left(V_{1}, A_{1}\right)$ and $D_{2}=\left(V_{2}, A_{2}\right)$ be digraphs with no common vertices. The direct sum of digraphs $D_{1}$ and $D_{2}$, denoted by $D_{1} \oplus D_{2}$, is the digraph with vertex and arc sets $V_{1} \cup V_{2}$ and $A_{1} \cup A_{2}$, respectively. In general, $\oplus_{i=1}^{k} D_{i}$ denote the direct sum of the digraphs $D_{1}=\left(V_{1}, A_{1}\right), \ldots, D_{k}=\left(V_{k}, A_{k}\right)$. If $D_{i}=D$ for all $i$, then we simply write $\oplus_{i=1}^{k} D_{i}=k D$. 


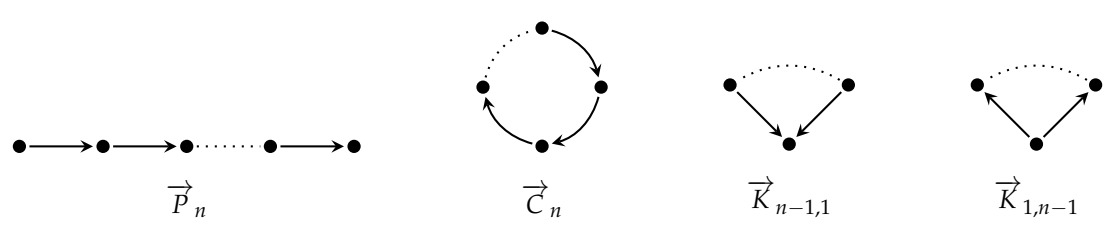

Figure 1. Orientations of $P_{n}, C_{n}$, and $S_{n}$.

The following notation and concepts were introduced in [1]. Let $D \in \mathcal{D}_{n}$. Let us denote by $n_{i}^{+}$(resp. $n_{i}^{-}$) the number of vertices in $D$ with out-degree (resp. in-degree) $i$, for all $0 \leq i \leq n-1$. For every $1 \leq i, j \leq n-1$, define the set

$$
A_{i j}=\left\{u v \in A: d_{u}^{+}=i \text { and } d_{v}^{-}=j\right\}
$$

The cardinality of $A_{i j}$ is denoted by $a_{i j}$. Clearly,

$$
\sum_{1 \leq i, j \leq n-1} a_{i j}=a \quad ; \quad \sum_{j=1}^{n-1} a_{i j}=i n_{i}^{+} \quad ; \quad \text { and } \quad \sum_{i=1}^{n-1} a_{i j}=j n_{j}^{-} \text {, }
$$

where $a$ is the number of $\operatorname{arcs} D$ has.

A VDB topological index is a function $\varphi$ induced by real numbers $\left\{\varphi_{i j}\right\}$, where $1 \leq i, j \leq n-1$, defined as [1]

$$
\varphi(D)=\frac{1}{2} \sum_{1 \leq i, j \leq n-1} a_{i j} \varphi_{i j}
$$

Equivalently,

$$
\varphi(D)=\frac{1}{2} \sum_{u v \in A} \varphi_{d_{u}^{+} d_{v}^{-}}
$$

When $\varphi_{i j}=\varphi_{j i}$ for all $1 \leq i, j \leq n-1$, we say that $\varphi$ is a symmetric VDB topological index. In this case, the expression given in (2) can be simplified. In fact, let

$$
p_{i j}=a_{i j}+a_{j i}
$$

for all $1 \leq i, j \leq n-1$, and

$$
p_{i i}=a_{i i}
$$

for all $i=1, \ldots, n-1$. Then

$$
\varphi(D)=\frac{1}{2} \sum_{(i, j) \in K} p_{i j} \varphi_{i j}
$$

where

$$
K=\{(i, j) \in \mathbb{N} \times \mathbb{N}: 1 \leq i \leq j \leq n-1\} .
$$

In particular, when $D=G$ is a graph, it was shown in [1] that Formula (6) reduces to

$$
\varphi(G)=\sum_{(i, j) \in K} m_{i j} \varphi_{i j}
$$

where $m_{i j}$ is the number of edges in $G$ which join vertices of degree $i$ and $j$. So we recover the degree-based-topological indices of graphs, a concept which has been, and currently is, extensively investigated in the mathematical and chemical literature [2-4]. For recent results, we refer to [5-12].

This paper is organized as follows. In Section 2, in a general setting (Theorem 1), we find sharp lower and upper bounds of a symmetric VDB topological index over the set $\mathcal{D}_{n}$. As a byproduct, we obtain over $\mathcal{D}_{n}$, sharp upper and lower bounds of well-known VDB topological indices, which include the First Zagreb index $\mathcal{M}_{1}\left(\varphi_{i j}=i+j\right)$ [13], the Second Zagreb index $\mathcal{M}_{2}\left(\varphi_{i j}=i j\right)$ [13], the Randić index $\chi\left(\varphi_{i j}=1 / \sqrt{i j}\right)$ [14], the Harmonic 
index $\mathcal{H}\left(\varphi_{i j}=2 /(i+j)\right)$ [15], the Geometric-Arithmetic $\mathcal{G A}\left(\varphi_{i j}=2 \sqrt{i j} /(i+j)\right)[16]$, the Sum-Connectivity $\mathcal{S C}\left(\varphi_{i j}=1 / \sqrt{i+j}\right)$ [17], the Atom-Bond-Connectivity $\mathcal{A B C}\left(\varphi_{i j}=\right.$ $\sqrt{(i+j-2) / i j})[18]$, and the Augmented Zagreb $\mathcal{A Z}\left(\varphi_{i j}=(i j /(i+j-2))^{3}\right)[19]$.

In Section 3, based on Theorem 2, we give sharp upper and lower bounds of symmetric VDB topological indices over the set $\mathcal{O T}(n)$, the set of oriented trees with $n$ vertices. In particular, we deduce sharp upper and lower bounds for the well-known indices mentioned above over $\mathcal{O} \mathcal{T}(n)$. Finally, in Section 4, we consider the problem of finding the extremal values of a symmetric VDB topological index among all orientations in $\mathcal{O}(G)$, the set of all orientations of a fixed graph $G$. In order to do this, we define strictly nondecreasing (resp. nonincreasing) symmetric VDB topological indices and show that for these indices, the value of any orientation at $G$ is not greater (resp. smaller) than half the value at $G$. Moreover, equality occurs, and only if the orientation is a sink-source orientation of $G$. In particular, when $G$ is a bipartite graph, we show that the sink-source orientations of $G$ attain extremal values.

\section{Bounds of VDB Topological Indices of Digraphs}

From now on, when we say that $\varphi$ is a symmetric VDB topological index, we mean that $\varphi$ is induced by the numbers $\left\{\varphi_{i j}\right\}$, where $(i, j) \in K$, and it is defined as in the equivalent definitions (2), (3), or (6). In the first part of this section, we generalize several results of [20] to digraphs.

Let $\varphi$ be a symmetric VDB topological index. Consider the function $f_{i j}=\frac{i j \varphi_{i j}}{i+j}$ defined over the set $K$. For each $(r, s) \in K$, consider the subset of $K$

$$
K_{r s}=\{(i, j) \in K:(i, j) \neq(r, s)\} .
$$

Recall that $q$ is the number of vertices which are sink or source vertices of a digraph $D$.

Lemma 1. Let $\varphi$ be a symmetric $V D B$ topological index and $D \in \mathcal{D}_{n}$. Let $(r, s) \in K$. Then

$$
2 \varphi(D)=(2 n-q) f_{r s}+\sum_{(i, j) \in K_{r s}}\left(f_{i j}-f_{r s}\right) \frac{i+j}{i j} p_{i j} .
$$

Proof. The numbers $\left\{p_{i j}\right\}$ defined in (4) in (5) satisfy the relation (see (10) in [1])

$$
\sum_{(i, j) \in K}\left(\frac{1}{i}+\frac{1}{j}\right) p_{i j}=2 n-\left(n_{0}^{+}+n_{0}^{-}\right) .
$$

Note that $q=n_{0}^{+}+n_{0}^{-}$. By (7),

$$
\frac{r+s}{r s} p_{r s}+\sum_{(i, j) \in K_{r s}}\left(\frac{1}{i}+\frac{1}{j}\right) p_{i j}=2 n-q,
$$

which implies

$$
p_{r s}=\frac{r s}{r+s}\left(2 n-q-\sum_{(i, j) \in K_{r s}}\left(\frac{1}{i}+\frac{1}{j}\right) p_{i j}\right) .
$$

On the other hand,

$$
\varphi(D)=\frac{1}{2} p_{r s} \varphi_{r s}+\frac{1}{2} \sum_{(i, j) \in K_{r s}} p_{i j} \varphi_{i j} .
$$


Now, substituting (8) in (9), we deduce

$$
\varphi(D)=\frac{1}{2} f_{r s}(2 n-q)+\frac{1}{2} \sum_{(i, j) \in K_{r s}} p_{i j} \frac{i+j}{i j}\left(f_{i j}-f_{r s}\right) .
$$

Let $\varphi$ be a symmetric VDB topological index with associated function $f_{i j}=\frac{i j \varphi_{i j}}{i+j}$. Define the sets

$$
K_{\min }(f)=\left\{(r, s) \in K: f_{r s}=\min _{(i, j) \in K} f_{i j}\right\},
$$

and

$$
K_{\max }(f)=\left\{(r, s) \in K: f_{r s}=\max _{(i, j) \in K} f_{i j}\right\} .
$$

We will denote by $K_{\min }^{c}(f)$ and $K_{\max }^{c}(f)$ the complements of $K_{\min }(f)$ and $K_{\max }(f)$ in $K$, respectively. We now generalize ([20], Theorem 2.3) to digraphs.

Theorem 1. Let $\varphi$ be a symmetric $V D B$ topological index and $D \in \mathcal{D}_{n}$. Then

$$
\frac{1}{2}(2 n-q) \min _{(i, j) \in K} f_{i j} \leq \varphi(D) \leq \frac{1}{2}(2 n-q) \max _{(i, j) \in K} f_{i j} .
$$

Moreover, equality on the left occurs, and only if $p_{x y}=0$ for all $(x, y) \in K_{\min }^{c}(f)$. Equality on the right occurs, and only if $p_{x y}=0$ for all $(x, y) \in K_{\max }^{c}(f)$.

Proof. Assume that $f_{r s}=\max _{(i, j) \in K} f_{i j}$, where $(r, s) \in K$. By Lemma 1 and the fact that $f_{i j} \leq$ $f_{r s}$ for all $(i, j) \in K$, we deduce

$$
\begin{aligned}
\varphi(D) & =\frac{1}{2}\left((2 n-q) f_{r s}+\sum_{(i, j) \in K_{r s}}\left(f_{i j}-f_{r s}\right) \frac{i+j}{i j} p_{i j}\right) \\
& \leq \frac{1}{2}(2 n-q) f_{r s} .
\end{aligned}
$$

On the other hand, since $\left(f_{i j}-f_{r s}\right) \frac{i+j}{i j} p_{i j}=0$ for all $(i, j) \in K_{\max }(f)$, it is clear that

$$
\sum_{(i, j) \in K_{r s}}\left(f_{i j}-f_{r s}\right) \frac{i+j}{i j} p_{i j}=0
$$

if, and only if $p_{x y}=0$ for all $(x, y) \in K_{\max }^{c}(f)$. By inequality (10), this is equivalent to $\varphi(D)=\frac{1}{2}\left(2 n-n_{0}^{+}-n_{0}^{-}\right) \max _{(i, j) \in K} f_{i j}$. The proof of the left inequality (and the equality condition) is similar.

So by Theorem 1, in order to find extremal values of a VDB topological index $\varphi$ over $\mathcal{D}_{n}$, we must find $K_{\min }(f)$ and $K_{\max }(f)$, where $f=\frac{i j \varphi_{i j}}{i+j}$. Fortunately, these were computed for the main VDB topological indices in [21] (see Table 1). 
Table 1. $K_{\min }(f)$ and $K_{\max }(f)$ for some VDB topological indices.

\begin{tabular}{ccccc}
\hline VDB Index & Notation & $\boldsymbol{\varphi}_{i j}$ & $\boldsymbol{K}_{\min }(f)$ & $\boldsymbol{K}_{\max }(f)$ \\
\hline First Zagreb [13] & $\mathcal{M}_{1}$ & $i+j$ & $(1,1)$ & $(n-1, n-1)$ \\
\hline Second Zagreb [13] & $\mathcal{M}_{2}$ & $i j$ & $(1,1)$ & $(n-1, n-1)$ \\
\hline Randić [14] & $\chi$ & $\frac{1}{\sqrt{i j}}$ & $(1, n-1)$ & $\{(i, j) \in K: i=j\}$ \\
\hline Harmonic [15] & $\mathcal{H}$ & $\frac{2}{i+j}$ & $(1, n-1)$ & $\{(i, j) \in K: i=j\}$ \\
\hline Geometric-Arithmetic [16] & $\mathcal{G A}$ & $\frac{2 \sqrt{i j}}{i+j}$ & $(1, n-1)$ & $(n-1, n-1)$ \\
\hline Sum-Connectivity [17] & $\mathcal{S C}$ & $\frac{1}{\sqrt{i+j}}$ & $(1, n-1)$ & $(n-1, n-1)$ \\
\hline Atom-Bond-Connectivity [18] & $\mathcal{A B C}$ & $\sqrt{\frac{i+j-2}{i j}}$ & $(1,1)$ & $(n-1, n-1)$ \\
\hline Augmented Zagreb [19] & $\mathcal{A Z}$ & $\left(\frac{i j}{i+j-2}\right)^{3}$ & $(1, n-1)$ & $(n-1, n-1)$ \\
\hline
\end{tabular}

An important class of digraphs which occur frequently as extremal values of VDB topological indices are the arc-balanced digraphs, which we define as follows.

Definition 1. A digraph $D$ is arc-balanced if $d_{u}^{+}=d_{v}^{-}$, for every arc $u v$ of $D$, and $q=0$.

A regular digraph is a digraph $D$ such that $d_{u}^{+}=d_{u}^{-}=r$, for all vertices $u$ in $D$, where $r$ is a positive integer. Clearly, every regular digraph is arc-balanced.

Example 1. The digraphs in Figure 2 are arc-balanced but not regular digraphs.
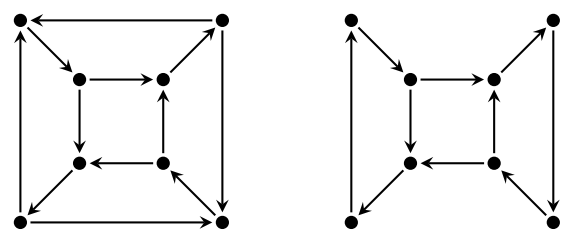

Figure 2. Arc-balanced digraphs.

Now we can give sharp upper and lower bounds for all VDB topological indices listed in Table 1. The following result is clear.

Lemma 2. Let $D \in \mathcal{D}_{n}$.

1. $p_{i j}=0$ for all $(i, j) \neq(1,1)$ if, and only if

$$
D=\bigoplus_{i=1}^{k_{1}} \vec{P}_{n_{i}} \oplus \bigoplus_{j=1}^{k_{2}} \vec{C}_{n_{j}},
$$

for some nonnegative integers $k_{1}$ and $k_{2}$.

2. $p_{i j}=0$ for all $(i, j) \in K$ such that $i<j$ and $q=0 \Leftrightarrow D$ is an arc-balanced digraph;

3. $p_{i j}=0$ for all $(i, j) \in K$ such that $(i, j) \neq(n-1, n-1) \Leftrightarrow D=K_{n}$;

4. $p_{i j}=0$ for all $(i, j) \neq(1, n-1)$ and $q=n \Leftrightarrow D=\vec{K}_{1, n-1}$ or $D=\vec{K}_{n-1,1}$;

5. $p_{i j}=0$ for all $(i, j) \neq(1,1)$ and $q=n \Leftrightarrow n$ is even and $D=\frac{n}{2} \vec{P}_{2}$.

6. $\quad p_{i j}=0$ for all $(i, j) \neq(1,1)$ and $q=n-1 \Leftrightarrow n$ is odd and $D=\frac{n-3}{2} \vec{P}_{2} \oplus \vec{P}_{3}$.

Lemma 3. Assume that $n$ is odd. Let $D \in \mathcal{D}_{n}$. If $q=n$, then $p_{11} \leq \frac{n-3}{2}$. 
Proof. Every vertex of $D$ is a sink vertex or a source vertex. Consequently,

$$
D=E \oplus p_{11} \vec{P}_{2}
$$

where $p_{11}(E)=0$. In particular,

$$
n=n(E)+2 p_{11}
$$

Since $n$ is odd, then $n(E)$ is also odd. Moreover, $n(E) \geq 3$, since $D$ has no isolated vertices. Hence,

$$
2 p_{11}=n-n(E) \leq n-3
$$

Corollary 1. Let $D \in \mathcal{D}_{n}$. Then

1.

$$
\left\lceil\frac{n}{2}\right\rceil \leq \mathcal{M}_{1}(D) \leq n(n-1)^{2}
$$

(a) Equality on the left occurs $\Leftrightarrow n$ is even and $D=\frac{n}{2} \vec{P}_{2}$ or $n$ is odd, and $D=$ $\frac{n-3}{2} \vec{P}_{2} \oplus \vec{P}_{3}$;

(b) Equality on the right occurs $\Leftrightarrow D=K_{n}$.

2.

$$
\left.\begin{array}{cc}
\frac{n}{4} & \text { if } n \text { even } \\
\frac{n+1}{4} & \text { if } n \text { odd }
\end{array}\right\} \leq \mathcal{M}_{2}(D) \leq \frac{1}{2} n(n-1)^{3} .
$$

(a) Equality on the left occurs $\Leftrightarrow n$ is even and $D=\frac{n}{2} \vec{P}_{2}$ or $n$ is odd and $D=$ $\frac{n-3}{2} \vec{P}_{2} \oplus \vec{P}_{3}$;

(b) Equality on the right occurs $\Leftrightarrow D=K_{n}$.

3.

$$
\frac{1}{2} \sqrt{n-1} \leq \chi(D) \leq \frac{n}{2}
$$

(a) Equality on the left occurs $\Leftrightarrow D=\vec{K}_{1, n-1}$ or $D=\vec{K}_{n-1,1}$;

(b) Equality on the right occurs $\Leftrightarrow D$ is an arc-balanced digraph.

4.

$$
\frac{n-1}{n} \leq \mathcal{H}(D) \leq \frac{n}{2}
$$

(a) Equality on the left occurs $\Leftrightarrow D=\vec{K}_{1, n-1}$ or $D=\vec{K}_{n-1,1}$;

(b) Equality on the right occurs $\Leftrightarrow D$ is an arc-balanced digraph.

5.

$$
\frac{(n-1)^{\frac{3}{2}}}{n} \leq \mathcal{G} \mathcal{A}(D) \leq \frac{n}{2 \sqrt[3]{(n-1)^{4}}}
$$

(a) Equality on the left occurs $\Leftrightarrow D=\vec{K}_{1, n-1}$ or $D=\vec{K}_{n-1,1}$;

(b) Equality on the right occurs $\Leftrightarrow D=K_{n}$.

6.

$$
\frac{n-1}{2 \sqrt{n}} \leq \mathcal{S C}(D) \leq \frac{1}{4} n \sqrt{2(n-1)}
$$

(a) Equality on the left occurs $\Leftrightarrow D=\vec{K}_{1, n-1}$ or $D=\vec{K}_{n-1,1}$;

(b) Equality on the right occurs $\Leftrightarrow D=K_{n}$. 
7.

$$
0 \leq \mathcal{A B C}(D) \leq \frac{n}{2} \sqrt{2(n-2)}
$$

(a) Equality on the left occurs $\Leftrightarrow D=\bigoplus_{i=1}^{k_{1}} \vec{P}_{n_{i}} \oplus \bigoplus_{j=1}^{k_{2}} \vec{C}_{n_{j}}$ for some nonnegative integers $k_{1}, k_{2}$.

(b) Equality on the right occurs $\Leftrightarrow D=K_{n}$.

8.

$$
\frac{1}{2} \frac{(n-1)^{4}}{(n-2)^{3}} \leq \mathcal{A Z}(D) \leq \frac{1}{16} n \frac{(n-1)^{7}}{(n-2)^{3}} .
$$

(a) Equality on the left occurs $\Leftrightarrow D=\vec{K}_{1, n-1}$ or $D=\vec{K}_{n-1,1}$;

(b) Equality on the right occurs $\Leftrightarrow D=K_{n}$.

Proof. Recall that $f_{i j}=\frac{i j \varphi_{i j}}{i+j}$ is the associated function of the symmetric VDB topological index $\varphi$. The expressions for $f_{i j}$ are shown in Table 2 .

Table 2. $f_{i j}$ for some VDB topological Indices.

\begin{tabular}{ccccccccc}
\hline VDB Index & $\mathcal{M}_{\mathbf{1}}$ & $\mathcal{M}_{\mathbf{2}}$ & $\chi$ & $\mathcal{H}$ & $\mathcal{G A}$ & $\mathcal{S C}$ & $\mathcal{A B C}$ & $\mathcal{A Z}$ \\
\hline \multirow{2}{*}{$f_{i j}$} & \multirow{2}{*}{$\frac{(i j)^{2}}{i+j}$} & $\frac{\sqrt{i j}}{i+j}$ & $\frac{2 i j}{(i+j)^{2}}$ & $\frac{2(i j)^{\frac{3}{2}}}{(i+j)^{2}}$ & $\frac{i j}{(i+j)^{\frac{3}{2}}}$ & $\frac{\sqrt{i j(i+j-2)}}{i+j}$ & $\frac{(i j)^{4}}{(i+j)(i+j-2)^{3}}$ \\
\hline
\end{tabular}

Since $0 \leq q \leq n$, we easily deduce the result from Theorem 1 and Lemma 2 .

We only have to separately consider $\mathcal{M}_{1}$ and $\mathcal{M}_{2}$ when $n$ is odd. By Theorem 1 ,

$$
2 \mathcal{M}_{1}(D) \geq 2 n-q \geq n \text {. }
$$

Since $n$ is odd, $2 \mathcal{M}_{1}(D)>n$, and so $2 \mathcal{M}_{1}(D) \geq n+1$. Equivalently,

$$
\mathcal{M}_{1}(D) \geq(n+1) / 2=\lceil n / 2\rceil .
$$

For the equality condition, it is clear that $\mathcal{M}_{1}\left(\frac{n-3}{2} \vec{P}_{2} \oplus \vec{P}_{3}\right)=\frac{n+1}{2}$. Conversely, suppose that $\mathcal{M}_{1}(D)=\frac{n+1}{2}$. Then by (11),

$$
n+1 \geq 2 n-q,
$$

which implies $q \geq n-1$. So there are only two possibilities: $q=n-1$ and $q=n$. If $q=n$, then by Lemma $3, p_{11} \leq \frac{n-3}{2}$. On the other hand, by Lemma 1 applied to $(r, s)=(1,2)$,

$$
\begin{aligned}
n+1= & 2 \mathcal{M}_{1}(D)=2 n+\sum_{(i, j) \neq(1,2)}(i j-2) \frac{i+j}{i j} p_{i j} \\
= & 2 n+(-1) 2 p_{11}+\sum_{\substack{i, j) \neq(1,2) \\
(i, j) \neq(1,1)}}(i j-2) \frac{i+j}{i j} p_{i j} .
\end{aligned}
$$

Thus,

$$
0 \leq \sum_{\substack{i, j) \neq(1,2) \\(i, j) \neq(1,1)}}(i j-2) \frac{i+j}{i j} p_{i j}=2 p_{11}-n+1
$$


which implies $p_{11} \geq \frac{n-1}{2}$, a contradiction. Hence, $q=n-1$. Consequently,

$$
\mathcal{M}_{1}(D)=\frac{n+1}{2}=\frac{1}{2}(2 n-q) .
$$

It follows from Theorem 1 that $p_{i j}=0$ for all $(i, j) \neq(1,1)$. Finally, by Lemma 2,

$$
D=\frac{n-3}{2} \vec{P}_{2} \oplus \vec{P}_{3}
$$

The case of $\mathcal{M}_{2}$ when $n$ is odd is similar.

In the case of the $\mathcal{A B C}$ index, note that $\varphi_{i j}=0$ if, and only if $(i, j)=(1,1)$. Then it is clear that

$$
\mathcal{A B C}\left(\bigoplus_{i=1}^{k_{1}} \vec{P}_{n_{i}} \oplus \bigoplus_{j=1}^{k_{2}} \vec{C}_{n_{j}}\right)=0 .
$$

Conversely, if $D$ is a digraph such that $0=\mathcal{A B C}(D)$, then

$$
0=\mathcal{A B C}(D)=\frac{1}{2} \sum_{(i, j) \in K} p_{i j} \varphi_{i j}=\frac{1}{2} \sum_{\substack{(i, j) \in K \\(i, j) \neq(1,1)}} p_{i j} \varphi_{i j},
$$

which implies $p_{i j}=0$ for all $(i, j) \neq(1,1)$. Hence, by part 1 of Lemma 2, $D=\bigoplus_{i=1}^{k_{1}} \vec{P}_{n_{i}} \oplus$ $\oplus_{j=1}^{k_{2}} \vec{C}_{n_{j}}$

Remark 1. Using a linear programming modeling technique, the authors in [22] find some of the extremal values given in Corollary 1.

Now we give bounds of VDB topological indices in terms of the number of arcs. Let $\varphi$ be a symmetric VDB topological index. Let us define

$$
L_{\max }=L_{\max }(\varphi)=\left\{(i, j) \in K: \varphi_{i j}=\max _{K} \varphi_{i j}\right\},
$$

and

$$
L_{\min }=L_{\min }(\varphi)=\left\{(i, j) \in K: \varphi_{i j}=\min _{K} \varphi_{i j}\right\} .
$$

The complements in $K$ are denoted by $L_{\max }^{c}$ and $L_{\min }^{c}$, respectively.

Theorem 2. Let $\varphi$ be a symmetric VDB topological index. If $D$ is a digraph with a arcs, then

$$
\frac{1}{2} a\left(\min _{K} \varphi_{i j}\right) \leq \varphi(D) \leq \frac{1}{2} a\left(\max _{K} \varphi_{i j}\right) .
$$

Equality on the left occurs if, and only if $p_{i j}=0$ for all $(i, j) \in L_{\min }^{c}$. Equality on the right occurs if, and only if $p_{i j}=0$ for all $(i, j) \in L_{\max }^{c}$.

Proof. From (2) and (1),

$$
\begin{gathered}
\varphi(D)=\frac{1}{2} \sum_{K} p_{i j} \varphi_{i j} \leq \frac{1}{2} \sum_{K} p_{i j} \max _{K} \varphi_{i j}=\frac{1}{2} a\left(\max _{K} \varphi_{i j}\right) . \\
\text { If } \varphi(D)=\frac{1}{2} a\left(\max _{K} \varphi_{i j}\right) \text {, then by (12) } \\
p_{i j}\left(\varphi_{i j}-\max _{K} \varphi_{i j}\right)=0,
\end{gathered}
$$


for all $(i, j) \in K$. Hence, if $(i, j) \in L_{\max }^{c}$, then $\varphi_{i j}-\max _{K} \varphi_{i j} \neq 0$ and so $p_{i j}=0$.

Conversely, if $p_{i j}=0$ for all $(i, j) \in L_{\max }^{c}$, then

$$
\begin{aligned}
\varphi(D) & =\frac{1}{2} \sum_{K} p_{i j} \varphi_{i j}=\frac{1}{2} \sum_{L_{\max }} p_{i j} \varphi_{i j}+\frac{1}{2} \sum_{L_{\max }^{c}} p_{i j} \varphi_{i j} \\
& =\frac{1}{2} \sum_{L_{\max }} p_{i j} \varphi_{i j}=\frac{1}{2} a\left(\max _{K} \varphi_{i j}\right) .
\end{aligned}
$$

The proof of the left inequality (and equality) is similar.

\section{Bounds of VDB Topological Indices of Tree Orientations}

The set of oriented trees with $n$ vertices is denoted by $\mathcal{O T}(n)$. It is our interest in this section to determine the extremal values of a VDB topological index over $\mathcal{O T}(n)$. Clearly, $a=n-1$ for every $T \in \mathcal{O} \mathcal{T}(n)$. Hence, by Theorem 2 we deduce the following.

Corollary 2. Let $T \in \mathcal{O} \mathcal{T}(n)$. Then

$$
\frac{1}{2}(n-1) \min _{K} \varphi_{i j} \leq \varphi(T) \leq \frac{1}{2}(n-1) \max _{K} \varphi_{i j} .
$$

Equality on the left occurs if, and only if $p_{i j}=0$ for all $(i, j) \in L_{\min }^{c}$. Equality on the right occurs if, and only if $p_{i j}=0$ for all $(i, j) \in L_{\max }^{c}$.

Now we can obtain a first list of sharp upper and lower bounds for some VDB topological indices over $\mathcal{O} \mathcal{T}(n)$.

Theorem 3. Let $T \in \mathcal{O} \mathcal{T}(n)$. Then

1. $\quad \frac{1}{2} \sqrt{n-1} \leq \chi(T) \leq \frac{n-1}{2} ;$

2. $\quad \frac{n-1}{n} \leq \mathcal{H}(T) \leq \frac{n-1}{2}$;

3. $\frac{(n-1)^{\frac{3}{2}}}{n} \leq \mathcal{G} \mathcal{A}(T) \leq \frac{n-1}{2}$;

4. $\quad \frac{n-1}{2 \sqrt{n}} \leq \mathcal{S C}(T) \leq \frac{\sqrt{2}}{4}(n-1)$;

5. $\quad \frac{1}{2} \frac{(n-1)^{4}}{(n-2)^{3}} \leq \mathcal{A Z}(T)$.

Moreover, equality on the left of $1-5$ occurs $\Leftrightarrow T=\vec{K}_{1, n-1}$ or $T=\vec{K}_{n-1,1}$. Equality on the right of $1-4$ occurs $\Leftrightarrow T=\vec{P}_{n}$.

Proof. The inequalities on the left (and equality conditions) are immediate consequence of Corollary 1 . The inequalities on the right of $1-4$ are consequence of Corollary 2 having in mind Table 3.

Table 3. $L_{\max }$ and $\max _{K} \varphi_{i j}$ for $\chi, \mathcal{H}, \mathcal{G A}$, and $\mathcal{S C}$.

\begin{tabular}{cccc}
\hline VDB Index & $\boldsymbol{\varphi}_{i j}$ & $\boldsymbol{L}_{\max }$ & $\max _{\boldsymbol{K}} \boldsymbol{\varphi}_{i j}$ \\
\hline$\chi$ & $\frac{1}{\sqrt{i j}}$ & $(1,1)$ & 1 \\
$\mathcal{H}$ & $\frac{2}{i+j}$ & $(1,1)$ & 1 \\
$\mathcal{G} \mathcal{A}$ & $\frac{2 \sqrt{i j}}{i+j}$ & $\{(i, j) \in K: i=j\}$ & 1 \\
$\mathcal{S C}$ & $\frac{1}{\sqrt{i+j}}$ & $(1,1)$ & $\frac{1}{\sqrt{2}}$ \\
\hline
\end{tabular}

We also use the fact that $T \in \mathcal{O} \mathcal{T}(n)$ is such that $p_{i j}=0$ for all $(i, j) \neq(1,1)$ if, and only if $T=\vec{P}_{n}$. Similarly, $p_{i j}=0$ for all $(i, j)$ such that $i<j$ if, and only if $T=\vec{P}_{n}$. 
Theorem 4. Let $T \in \mathcal{O} \mathcal{T}(n)$. Then

1. $0 \leq \mathcal{A B C}(T) \leq \frac{1}{2} \sqrt{(n-1)(n-2)}$;

2. $(n-1) \leq \mathcal{M}_{1}(T)$;

3. $\quad \frac{1}{2}(n-1) \leq \mathcal{M}_{2}(T)$.

Moreover, equality on the left of 1-3 occurs $\Leftrightarrow T=\vec{P}_{n}$. Equality on the right of 1 occurs $\Leftrightarrow T=\vec{K}_{1, n-1}$ or $T=\vec{K}_{n-1,1}$.

Proof. The inequalities on the left of 1-3 (and equality conditions) are a consequence of Corollary 2, having in mind Table 4.

Table 4. $L_{\min }$ and $\min _{K} \varphi_{i j}$ for $\mathcal{A B C}, \mathcal{M}_{1}$, and $\mathcal{M}_{2}$.

\begin{tabular}{cccc}
\hline VDB Index & $\varphi_{i j}$ & $L_{\text {min }}$ & $\min _{K} \varphi_{i j}$ \\
\hline $\mathcal{A B C}$ & $\sqrt{\frac{i+j-2}{i j}}$ & $(1,1)$ & 0 \\
$\mathcal{M}_{1}$ & $i+j$ & $(1,1)$ & 2 \\
$\mathcal{M}_{2}$ & $i j$ & $(1,1)$ & 1 \\
\hline
\end{tabular}

And the fact that $T \in \mathcal{O} \mathcal{T}(n)$ is such that $p_{i j}=0$ for all $(i, j) \neq(1,1)$ if, and only if $T=\vec{P}_{n}$. On the other hand, the right inequality in 1 holds again by Corollary 2 , bearing in mind Table 5.

Table 5. $L_{\max }$ and $\max _{K} \varphi_{i j}$ for $\mathcal{A B C}$.

\begin{tabular}{cccc}
\hline VDB Index & $\varphi_{i j}$ & $L_{\max }$ & $\max _{K} \varphi_{i j}$ \\
\hline $\mathcal{A B C}$ & $\sqrt{\frac{i+j-2}{i j}}$ & $(1, n-1)$ & $\sqrt{\frac{n-2}{n-1}}$ \\
\hline
\end{tabular}

And the fact that $T \in \mathcal{O} \mathcal{T}(n)$ is such that $p_{i j}=0$ for all $(i, j) \neq(1, n-1)$ if, and only if $T=\vec{K}_{1, n-1}$ or $T=\vec{K}_{n-1,1}$.

The only extremal values we have not determined yet are the maximal values of $\mathcal{M}_{1}, \mathcal{M}_{2}$, and $\mathcal{A Z}$ over $\mathcal{O} \mathcal{T}(n)$. The problem in these indices is that $L_{\max }=(n-1, n-1)$, and there is no oriented tree such that $p_{i j}=0$ for all $(i, j) \neq(n-1, n-1)$. In the next section we will show that the maximum value of $\mathcal{M}_{1}$ and $\mathcal{M}_{2}$ over $\mathcal{O T}(n)$ is attained in $\vec{K}_{1, n-1}$ or $\vec{K}_{n-1,1}$ (see Theorem 6 ). We propose the following problem.

Problem 1. Find the maximum value of $\mathcal{A Z}$ over $\mathcal{O} \mathcal{T}(n)$.

\section{Bounds of VDB Topological Indices over Orientations of a Fixed Graph}

Let $\varphi$ be a symmetric VDB topological index and $G$ a graph. Let $\mathcal{O}(G)$ be the set of orientations of the graph $G$. Our main concern now is to determine the extremal values of a symmetric VDB topological index over $\mathcal{O}(G)$. In order to do this, let us define a partial order over $K$ as follows: if $(i, j),(k, l) \in K$, then

$$
(i, j) \preceq(k, l) \Leftrightarrow i \leq k \text { and } j \leq l .
$$

Definition 2. Let $\varphi$ be a symmetric VDB topological index. We say that $\varphi$ is nondecreasing (resp. nonincreasing) over $K$, if for every $(i, j),(k, l) \in K$ :

$$
(i, j) \preceq(k, l) \Rightarrow \varphi_{i j} \leq \varphi_{k l}\left(\operatorname{resp} . \varphi_{i j} \geq \varphi_{k l}\right) .
$$


Furthermore, if for every $(i, j),(k, l) \in K$ :

$$
(i, j) \preceq(k, l) \text { and } \varphi_{i j}=\varphi_{k l} \Rightarrow(i, j)=(k, l),
$$

we will say that $\varphi$ is strictly nondecreasing (resp. strictly nonincreasing).

Example 2. Consider the generalized Randic index $\chi_{\alpha}$ induced by the numbers $(i j)^{\alpha}$, where $\alpha \in \mathbb{R}$, $\alpha \neq 0$. Clearly, $\chi_{\alpha}$ is strictly nondecreasing when $\alpha>0$, and strictly nonincreasing when $\alpha<0$. In particular, the Randic index $\chi$ is strictly nonincreasing and the second Zagreb index $\mathcal{M}_{2}$ is strictly nondecreasing. Additionally, the harmonic index and the sum-connectivity index are strictly nonincreasing, and the first Zagreb $\mathcal{M}_{1}$ is strictly nondecreasing.

Theorem 5. Let $\varphi$ be a strictly nondecreasing (resp. nonincreasing) symmetric VDB topological index and $G$ a graph. Let $D$ be any orientation of $G$. Then

$$
\varphi(D) \leq \frac{1}{2} \varphi(G)\left(\operatorname{resp} . \varphi(D) \geq \frac{1}{2} \varphi(G)\right) .
$$

Equality holds if, and only if $D$ is a sink-source orientation of $G$.

Proof. We will assume that $\varphi$ is strictly nondecreasing, and the other case is similar. Note that

$$
d_{u}=d_{u}^{+}+d_{u}^{-}
$$

for every vertex $u$ of $G$. Hence, for any arc $u v$ of $D,\left(d_{u}^{+}, d_{v}^{-}\right) \preceq\left(d_{u}, d_{v}\right)$. It follows by the nondecreasing property of $\varphi$ and (3),

$$
\varphi(D)=\frac{1}{2} \sum_{u v \in A} \varphi_{d_{u}^{+} d_{v}^{-}} \leq \frac{1}{2} \sum_{u v \in G} \varphi_{d_{u} d_{v}}=\frac{1}{2} \varphi(G) .
$$

If $D$ is a sink-source orientation of $G$, then $d_{u}^{+}=0$ or $d_{u}^{-}=0$, for all vertices $u$ of $V$. If $v w$ is an arc of $D$ then $d_{v}^{+} \neq 0$ and $d_{w}^{-} \neq 0$. Hence, $d_{v}^{-}=0$ and $d_{w}^{+}=0$, which implies by (13) that $d_{v}=d_{v}^{+}$and $d_{w}=d_{w}^{-}$. Hence,

$$
\varphi(D)=\frac{1}{2} \sum_{u v \in A} \varphi_{d_{u}^{+} d_{v}^{-}}=\frac{1}{2} \sum_{u v \in G} \varphi_{d_{u} d_{v}}=\frac{1}{2} \varphi(G) .
$$

Conversely, assume that $\varphi(D)=\frac{1}{2} \varphi(G)$. Then by (14), for every $u v \in A$

$$
\left(d_{u}^{+}, d_{v}^{-}\right) \preceq\left(d_{u}, d_{v}\right) \text { and } \varphi_{d_{u}^{+} d_{v}^{-}}=\varphi_{d_{u} d_{v}} .
$$

Now since $\varphi$ is strictly nondecreasing, $\left(d_{u}^{+}, d_{v}^{-}\right)=\left(d_{u}, d_{v}\right)$ for every $u v \in A$. Finally, by (13), $d_{u}^{-}=0$ and $d_{v}^{+}=0$. This clearly implies that $D$ is a sink-source orientation of G.

Corollary 3. Let $\varphi$ be a strictly nondecreasing (resp. nonincreasing) symmetric VDB topological index and $G$ a bipartite graph. Then the maximal (resp. minimal) value of $\varphi$ over $\mathcal{O}(G)$ is attained in a sink-source orientation of $G$.

Proof. We assume that $\varphi$ is strictly nondecreasing, and the other case is similar. Since $G$ is a bipartite graph, $G$ has a sink-source orientation which we call $E$ [23]. Let $D$ be any orientation of $G$. Then by Theorem 5,

$$
\varphi(E)=\frac{1}{2} \varphi(G) \geq \varphi(D) .
$$


Example 3. Consider the path tree $P_{n}$. By Example 2 and Corollary 3, the sink-source orientation $E \in \mathcal{O}\left(P_{n}\right)$ depicted in Figure 3 attains the maximal value for $\mathcal{M}_{1}, \mathcal{M}_{2}$ and $\chi_{\alpha}$ when $\alpha>0$, over $\mathcal{O}\left(P_{n}\right)$. On the other hand, E attains the minimal value of $\mathcal{H}, \mathcal{S C}$ and $\chi_{\alpha}$ when $\alpha<0$, over $\mathcal{O}\left(P_{n}\right)$.

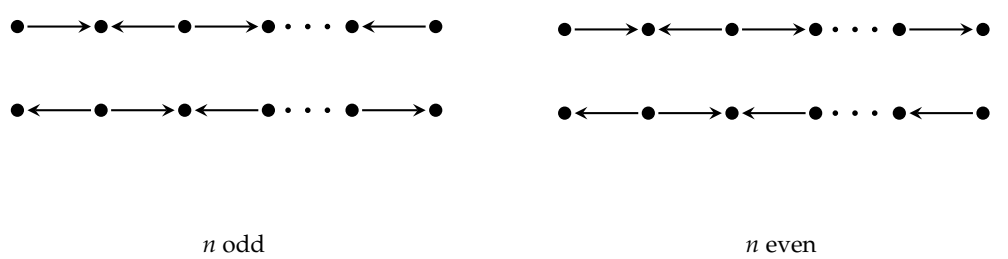

Figure 3. Sink-source orientations of $P_{n}$.

Example 4. In [24] the authors studied the extreme values of $\chi$ on the set of all the orientations of hexagonal chains with $k$ hexagons.

Theorem 6. Let $T \in \mathcal{O} \mathcal{T}(n)$. Then

1. $\mathcal{M}_{1}(T) \leqslant \frac{1}{2} n(n-1)$;

2. $\mathcal{M}_{2}(T) \leqslant \frac{1}{2}(n-1)^{2}$.

Moreover, equalities $1-2$ occur $\Leftrightarrow T=\vec{K}_{1, n-1}$ or $T=\vec{K}_{n-1,1}$.

Proof. Let $G$ be a tree of order $n$. If $G$ is different from $S_{n}$, then [25]

$$
\begin{aligned}
& \mathcal{M}_{1}(G)<\mathcal{M}_{1}\left(S_{n}\right)=n(n-1) \\
& \mathcal{M}_{2}(G)<\mathcal{M}_{2}\left(S_{n}\right)=(n-1)^{2} .
\end{aligned}
$$

Let $T \in \mathcal{O} \mathcal{T}(n)$ and suppose that $T$ is an orientation of a tree $G$. By Theorem 5 and the above equation,

$$
\begin{aligned}
& \mathcal{M}_{1}(T) \leqslant \frac{1}{2} \mathcal{M}_{1}(G) \leqslant \frac{1}{2} n(n-1) \\
& \mathcal{M}_{2}(T) \leqslant \frac{1}{2} \mathcal{M}_{2}(G) \leqslant \frac{1}{2}(n-1)^{2} .
\end{aligned}
$$

Equality occurs if, and only if $T$ is a sink-source orientation of $S_{n}$, in other words, $T=\vec{K}_{1, n-1}$ or $T=\vec{K}_{n-1,1}$.

Author Contributions: The two authors have contributed equally to the article. Both authors have read and agreed to the published version of the manuscript.

Funding: This research received no external funding.

Institutional Review Board Statement: Not applicable.

Informed Consent Statement: Not applicable.

Data Availability Statement: Not applicable.

Conflicts of Interest: The authors declare no conflict of interest.

\section{References}

1. Monsalve, J.; Rada, J. Vertex-degree-based topological indices of digraphs. Discrete Appl. Math. 2021, 295, 13-24. [CrossRef]

2. Devillers, J.; Balaban, A.T. (Eds.) Topological Indices and Related Descriptors in QSAR and QSPR; Gordon \& Breach: Amsterdam, The Netherlands, 1999.

3. Gutman, I. Degree-based topological indices. Croat. Chem. Acta 2013, 86, 351-361. [CrossRef]

4. Todeschini, R.; Consonni, V. Handbook of Molecular Descriptors; Wiley-VCH: Weinheim, Germany, 2000.

5. Ali, A.; Furtula, B.; Gutman, I.; Vukičević, D. Augmented Zagreb index: Extremal results and bounds. MATCH Commun. Math. Comput. Chem. 2021, 85, 211-244. 
6. Cui, S.Y.; Wang, W.; Tian, G.X.; Wu, B. On the arithmetic-geometric index of graphs. MATCH Commun. Math. Comput. Chem. 2021, 85, 87-107.

7. Das, K.C.; Rodríguez, J.M.; Sigarreta, J.M. On the maximal general ABC index of graphs with given maximum degree. Appl. Math. Comput. 2020, 386, 125531. [CrossRef]

8. Gutman, I. Geometric approach to degree based topological indices: Sombor indices. MATCH Commun. Math. Comput. Chem. 2021, 86, 11-16.

9. Horoldagva, B.; Xu, C. On Sombor index of graphs. MATCH Commun. Math. Comput. Chem. 2021, 86, 703-713.

10. Molina, E.D.; Rodríguez, J.M.; Sánchez, J.L.; Sigarreta, J.M. Some Properties of the Arithmetic-Geometric Index. Symmetry 2021, 13, 857. [CrossRef]

11. Rodríguez, J.M.; Sánchez, J.L.; Sigarreta, J.M.; Tourís, E. Bounds on the arithmetic-geometric index. Symmetry 2021, $13,689$. [CrossRef]

12. Sigarreta, J.M. Mathematical Properties of Variable Topological Indices. Symmetry 2021, 13, 43. [CrossRef]

13. Gutman, I.; Trinajstić, N. Graph theory and molecular orbitals. Total $\pi$-electron energy of alternant hydrocarbons. Chem. Phys. Lett. 1972, 17, 535-538. [CrossRef]

14. Randić, M. On characterization of molecular branching. J. Am. Chem. Soc. 1975, 97, 6609-6615. [CrossRef]

15. Zhou, B.; Trinajstić, N. On a novel connectivity index. J. Math. Chem. 2009, 6, 1252-1270. [CrossRef]

16. Vukičević, D.; Furtula, B. Topological index based on the ratios of geometrical and arithmetical means of end-vertex degrees of edges. J. Math. Chem. 2009, 46, 1369-1376. [CrossRef]

17. Zhong, L. The harmonic index for graphs. Appl. Math. Lett. 2012, 25, 561-566. [CrossRef]

18. Estrada, E.; Torres, L.; Rodríguez, L.; Gutman, I. An atom-bond connectivity index: Modelling the enthalpy of formation of alkanes. Indian J. Chem. 1998, 37A, 849-855.

19. Furtula, B.; Graovac, A.; Vukičević, D. Augmented Zagreb index. J. Math. Chem. 2010, 48, 370-380. [CrossRef]

20. Rada, J.; Cruz, R. Vertex-degree-based topological indices over graphs. MATCH Commun. Math. Comput. Chem. 2014, 72, 603-616.

21. Cruz, R.; Pérez, T.; Rada, J. Extremal values of vertex-degree-based topological indices over graphs. J. Appl. Math. Comput. 2015, 48, 395-406. [CrossRef]

22. Deng, H.; Yang, J.; Tang, Z.; Yang, J.; You M. On the vertex-degree based invariants of digraphs. arXiv 2021, arXiv:2104.14742.

23. Monsalve, J.; Rada, J. Oriented bipartite graphs with minimal trace norm. Linear Multilinear Algebra 2019, 67, 1121-1131. [CrossRef]

24. Bermudo, S.; Monsalve, J.; Rada, J. Orientations of hexagonal chains with extremal values of the Randić index. Int. J. Quantum Chem. 2021, 121, e26744. [CrossRef]

25. Deng, H. A unified approach to the extremal Zagreb indices for trees, unicyclic graphs and bicyclic graphs. MATCH Commun. Math. Comput. Chem. 2007, 57, 597-616. 